\title{
Prospective study of the relationship between coffee and tea with colorectal cancer risk: The PLCO Cancer Screening Trial
}

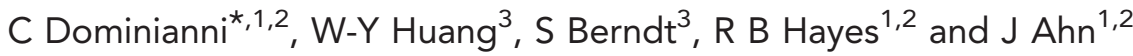

${ }^{1}$ Division of Epidemiology, Department of Population Health, New York University School of Medicine, 650 First Avenue, New York, NY 10016, USA; ${ }^{2}$ New York University Cancer Institute, 522 First Avenue, New York, NY 10016, USA and ${ }^{3}$ Division of Cancer Epidemiology and Genetics, National Cancer Institute, 6120 Executive Boulevard, Bethesda, MD 20892, USA

Background: Coffee and tea are commonly consumed and carry potential anticancer components that could reduce the risk of colorectal cancer; however, their relationships with colorectal cancer risk remain inconsistent.

Methods: A prospective analysis was carried out to examine the relationships of coffee and tea intake with colorectal cancer risk in 57398 men and women in the intervention arm of the National Cancer Institute-Prostate, Lung, Colorectal and Ovarian Cancer Screening Trial, a national screening study that limits differential detection biases. Coffee and tea intakes were assessed by food frequency questionnaire.

Results: Six hundred and eighty-one incident colorectal cancer cases were ascertained during a median follow-up of 11.4 years. Greater coffee intake was not associated with risk of colorectal cancer (relative risk $(R R)=1.08,95 \%$ confidence interval $(C l)=0.79-1.48$, $\left.P_{\text {trend }}=0.23\right)$. Stratifying by cancer site $\left(P_{\text {heterogeneity }}=0.48\right)$ or stage $\left(P_{\text {heterogeneity }}=0.83\right)$ did not alter the relationship. Associations remained unchanged in subsets of participants for either caffeinated or decaffeinated coffee or when stratifying by several colorectal cancer risk factors. Similarly, greater tea intake was not associated with colorectal cancer risk overall (RR $=0.77,95 \%$ $\left.\mathrm{Cl}=0.55-1.09, P_{\text {trend }}=0.17\right)$ or by cancer site $\left(P_{\text {heterogeneity }}=0.14\right)$ or stage $\left(P_{\text {heterogeneity }}=0.60\right)$. These associations were not modified by several colorectal cancer risk factors.

Conclusion: The findings of this study do not provide evidence to suggest that drinking coffee or tea is beneficial in protecting against colorectal cancer.

Colorectal cancer (CRC) is the third most common cancer and the third leading cause of cancer death in the United States (American Cancer Society, 2011). Much attention has been focused on coffee and tea consumption and CRC because these beverages are widely consumed and are rich sources of anticancer components. Numerous in vitro and in vivo studies have suggested that coffee constituents, such as caffeine and chlorogenic acid, have a role in reducing colonic tumor formation (Merighi et al, 2007; Bernstein et al, 2011; Kang et al, 2011; Bessler et al, 2012). Similarly, ( - )-epigallocatechin-3-gallate (EGCG), the major catechin found in green tea and black tea polyphenols have been shown to have chemoprotective properties in in vitro and in in vivo studies (Xu et al, 1996; Sengupta et al, 2006; Patel et al, 2008; Shimizu et al, 2008; Sukhthankar et al, 2010).

The relationship of coffee and tea consumption to CRC has been examined in previous epidemiologic studies with inconsistent findings. Most case-control studies have reported an inverse relationship of coffee and tea consumption to CRC (Galeone et al, 2010; Li et al, 2012). Recently, a large cohort study has also reported an inverse association of coffee with CRC (Sinha et al, 2012). However, most cohort studies have reported a null association between coffee and CRC risk (Je et al, 2009; Li et al, 2012). 
For tea, results have also been inconsistent across various studies (Sun et al, 2006).

We conducted a prospective cohort study to examine the relationship of coffee and tea intake with the risk of CRC in 57398 men and women in the National Cancer Institute-Prostate, Lung, Colorectal and Ovarian (PLCO) Cancer Screening Trial (Prorok et al, 2000), a national screening-based study that limits biases due to differential detection of cancers. Subjects in our study were randomised to flexible sigmoidoscopy screening and were subsequently followed to examine the relationship of coffee and tea consumption to CRC risk, overall and by tumor site and stage.

\section{SUBJECTS AND METHODS}

Study population. The PLCO Cancer Screening Trial is a multicentre trial designed to evaluate screening methods for the early detection of several cancers (Weissfeld et al, 2005). Briefly, 154900 men and women, aged 55-74 years, were recruited from 10 centres (Birmingham, AL; Denver, CO; Detroit, MI; Honolulu, HI; Marshfield, WI; Minneapolis, MN; Pittsburgh, PA; Salt Lake City, UT; St Louis, MO; and Washington, DC, USA) between 1993 and 2001 and randomised to receive either CRC screening (sigmoidoscopy, insertion to at least $50 \mathrm{~cm}$ with $>90 \%$ of mucosa visible or a suspect lesion identified) or standard care. Participants completed baseline and diet questionnaires. The study was approved by the institutional review boards at the National Cancer Institute and the screening centres, and all participants provided informed consent.

Of the 75187 men and women who were randomly assigned to the intervention arm of the trial, 58449 participants (78\%) completed both the baseline risk factor and food frequency $(<8$ questions missing) questionnaires. We further excluded 1051 participants for the following reasons: a missing response for coffee intake $(n=195)$; previous history of cancer, except basal-cell skin cancer $(n=2758)$; or self-reported history of ulcerative colitis, Crohn's disease, familial polyposis or Gardner's syndrome $(n=1140)$. After exclusions, the analytic cohort comprised 57398 subjects (some participants were in more than one exclusion category). Within this cohort, 47678 (83\%) received a complete flexible sigmoidoscopy screening at baseline.

Colorectal cancer ascertainment. As part of the CRC screening portion of the PLCO trial, flexible sigmoidoscopic visualisation of the distal colon was carried out at baseline and then again at 3 or 5 years (protocol changed from rescreening at 3 years to 5 years in April 1995). Those who had suspected lesions for colorectal neoplasia (i.e., sigmoidoscopically visualised polypoid lesions or masses) were referred to their health-care providers for endoscopic and histopathologic follow-up. Subjects were also sent annual questionnaires asking about recent cancer diagnoses. The PLCO trial obtained all available medical and pathological reports on all lesions removed during the diagnostic endoscopy and related surgical procedures for all subjects with an abnormal sigmoidoscopic examination, or all colorectal cancers reported on the questionnaires or through death certificates. This information was abstracted and coded by trained medical abstractors. Only histologically confirmed cases were included in the analysis.

Coffee and tea intake. At study baseline, participants completed a Baseline Questionnaire on sociodemographics, smoking status, lifestyle factors, and personal and family medical history. Dietary intake was assessed by a Dietary Questionnaire (DQX) given at baseline. This food frequency questionnaire included 137 individual food items, 77 of which queried about usual portion size and frequency (https://www.plcostars.com/Public/Documents/PLCO/DQX.pdf). The DQX was designed on two previously validated food frequency questionnaires (Willett et al, 1985; Block et al, 1986).
Nutrient and food group values were calculated by the method developed by Subar and co-workers (Subar et al, 2000), using US dietary data and the pyramid food group servings database from the US Department of Agriculture (CSFII) (1997). The categorical groupings of the coffee and tea variables were derived from the combination of the number of servings and serving size. In addition, caffeinated and decaffeinated coffee intake was assessed by a second dietary questionnaire, the NIH Health Diet History Questionnaire (DHQ) (https://www.plcostars.com/ Public/Documents/PLCO/DHQ.pdf), which was administered to screening arm subjects in about the third year of follow-up, beginning in December 1998. The correlations between the two diet questionnaires were $r=0.63$ and 0.61 for coffee and tea intakes, respectively. Coffee intake was assigned into one of five categories for each questionnaire: none (reference group), $<1$ cup per day, 1 cup per day, $2-3$ cups per day and $\geqslant 4$ cups per day. Tea intake was assigned to one of four categories: none (reference group), $<1$ cup per week, 1 cup per day and $\geqslant 2$ cups per day.

Statistical analysis. We estimated relative risks (RRs) and 95\% confidence intervals (CIs) with Cox proportional hazards models (Cox, 1972). We calculated person-years of follow-up time from the date of the baseline DQX until the date of CRC diagnosis, death or end of follow-up, whichever occurred first. We confirmed that the proportional hazard assumption was met for the main exposures by visualisation of Schoenfeld residuals' plots. Multivariable analyses were adjusted for potentially confounding factors, including age (continuous), gender (male, female), race (White, Black, Asian, other), PLCO study centre, family history of CRC (first degree; yes, no), smoking status (never, current, former, pipe/ cigar only), education ( $<12$ years, 12 years of high school, post high school or college, and college graduation or more), history of diabetes (yes, no), body mass index (BMI) (18.5-24.9, 25.0-29.9 and $30.0 \mathrm{~kg} \mathrm{~m}^{-2}$ or more), number of screenings 3 years before the trial (none, once and more than once), physical activity (none, $<1$, 1, 2, 3 and $4+\mathrm{h}$ per week), non-steroidal anti-inflammatory drug intake (none, aspirin only, ibuprofen only, both aspirin and ibuprofen), hormone status among women (never, former and current), meat intake (g per day), fruit intake (servings per day), vegetable intake (servings per day) and alcohol intake (g per day). Further adjustment of total energy intake did not significantly modify risk estimates, so it was not included in analyses. Missing values were generally imputed into the most common category. To control for any confounding effect that tea might have on coffee or vice versa, tea and coffee variables were additionally included in the respective models. Tests for linear trends were conducted by assigning the median value for each category and treating that term as a single continuous variable in the model. In a subanalysis, we excluded the first 3 years of follow-up to minimse effects of preclinical disease. To reduce the effect of flexible sigmoidoscopy screening, analyses were also carried out in only those who received screening at baseline. Stratified analyses were carried out to examine possible interactions between coffee or tea and the key risk factors of age, gender, smoking status, BMI, meat intake and alcohol intake. We formally tested for interactions using loglikelihood ratio tests. All statistical tests were two-sided and considered significant at $P<0.05$.

As a subanalysis, caffeinated and decaffeinated coffee analyses were carried out in subsets of participants who had completed coffee-related questions in the additional DHQ questionnaire. Caffeinated coffee intake was defined as those who only consumed caffeinated coffee $(n=29099)$ and decaffeinated coffee intake was defined as those who only consumed decaffeinated coffee $(n=18262)$. Those who consumed both caffeinated and decaffeinated coffees were not included in either analysis. Person-years of follow-up time were calculated from the date of completing the DHQ until the date of CRC diagnosis, death or end of follow-up, 
whichever occurred first. All analyses for the DQX and DHQ were carried out using SAS, version 9.3 (SAS Institute, Cary, NC, USA).

\section{RESULTS}

Baseline characteristics. During the median follow-up time of 11.4 years, 587683 person-years were accumulated and 681 incident CRC cases were identified, including 381 proximal and 148 distal colon cancers and 142 rectal cancers. By stage, 235 cases were diagnosed as stage I, 161 cases were diagnosed as stage II and 249 cases were diagnosed as advanced (stage III/IV).

Eighty-eight percent of the population consumed coffee; the overall median coffee consumption was 1 cup per day. Greater coffee consumption was related to being male, a current smoker, being less physically active, a more frequent consumer of aspirin, meat and alcohol and a less frequent consumer of fruits and vegetables (Table 1). Among women, greater coffee consumption was related to less use of postmenopausal hormones. Greater tea consumers were more likely to be women, use aspirin less and consume more fruits and vegetables but less alcohol. Among women, higher tea consumers were more likely to have used postmenopausal hormones.

Coffee intake and colorectal cancer risk. In the age-adjusted model, greater coffee intake was not associated with risk of CRC (Table 2). The association remained unchanged after adjusting for non-dietary risk factors and in the final full model, which further adjusted for meat, fruit and vegetable intake, the association remained null ( $\mathrm{RR}$ for $\geqslant 4$ cups per day $v$ s none $=1.08,95 \%$ $\mathrm{CI}=0.79-1.48, P_{\text {trend }}=0.23$ ). In addition, adjusting for tea intake also did not affect the association $(\mathrm{RR}=1.06,95 \% \mathrm{CI}=0.77-1.46$, $\left.P_{\text {trend }}=0.26\right)$. When we excluded the first 3 years of follow-up, this relationship remained unchanged $(\mathrm{RR}=1.09,95 \% \mathrm{CI}=0.72-1.64$, $\left.P_{\text {trend }}=0.75\right)$. Excluding participants who did not receive complete flexible sigmoidoscopy screening also did not alter the relationship $\left(\mathrm{RR}=1.09,95 \% \mathrm{CI}=0.78-1.54, P_{\text {trend }}=0.19\right)$. Both caffeinated and decaffeinated coffees were not associated with CRC risk (for caffeinated coffee: $\mathrm{RR}=1.08,95 \% \mathrm{CI}=0.67-1.75$; for decaffeinated coffee: $\mathrm{RR}=1.53,95 \% \mathrm{CI}=0.75-3.09$ ). Restricting the analysis to individuals who had the same responses in both the baseline DQX and the follow-up DHQ did not alter the relationship between coffee and CRC risk (data not shown).

These associations were consistently null by cancer site (for proximal: $\mathrm{RR}=1.33,95 \% \mathrm{CI}=0.86-2.05$; for distal: $\mathrm{RR}=1.11$, $95 \% \mathrm{CI}=0.58-2.13$; and for rectal: $\mathrm{RR}=0.72,95 \% \mathrm{CI}=0.36-1.44$; $P$ for heterogeneity $=0.48$ ) and stage (I/II and III/IV, $P$ for heterogeneity $=0.83$ ) (Table 2 ). Limiting our analysis to the $93.1 \%$ colorectal adenocarcinoma cases did not alter the relationship of coffee intake and CRC risk. The association between coffee intake and risk of CRC was not significantly modified by gender $(P$ for interaction $=0.57$ ) or age at baseline ( $P$ for interaction $=0.25$ ). We also found no statistically significant differences in the association between coffee and CRC risk by BMI ( $P$ for interaction $=0.87)$, smoking status $(P$ for interaction $=0.64)$, alcohol consumption $(P$ for interaction $=0.62)$ or meat intake $(P$ for interaction $=0.30)$.

Tea intake and colorectal cancer risk. As shown in Table 3, there was a significant inverse association between the highest quartile of tea intake and $\mathrm{CRC}$ risk $(\mathrm{RR}=0.66,95 \% \mathrm{CI}=0.47-0.92$, $\left.P_{\text {trend }}=0.02\right)$ in the age-adjusted model; however, the association was not statistically significant after adjusting for non-dietary factors and after full adjustment $(\mathrm{RR}=0.77,95 \% \mathrm{CI}=0.55-1.09$, $\left.P_{\text {trend }}=0.17\right)$. Additionally, including coffee intake into the model did not change the relationship $(\mathrm{RR}=0.78,95 \% \mathrm{CI}=0.55-1.11$, $P_{\text {trend }}=0.19$ ). Further excluding the first 3 years of follow-up did not affect the relationship $(\mathrm{RR}=0.85,95 \% \mathrm{CI}=0.55-1.31$, $\left.P_{\text {trend }}=0.81\right)$. The association remained nonsignificant after exclusion of participants who did not receive complete flexible sigmoidoscopy $\left(\mathrm{RR}=0.83,95 \% \mathrm{CI}=0.56-1.21, P_{\text {trend }}=0.28\right)$.

As with coffee, subanalysis by cancer site (proximal, distal and rectal, $P$ for heterogeneity $=0.14)$ and stage $(\mathrm{I} / \mathrm{II}, \mathrm{III} / \mathrm{IV}, P$ for heterogeneity $=0.60)$ did not reveal significant associations between tea and CRC risk (Table 3). The association remained null with the analysis of adenocarcinoma cases only (93.1\%). Similar nonsignificant associations were observed for men and women $(P$ for interaction $=0.99)$ and for younger $(\leqslant 62$ years $)$ and older participants ( $>62$ years; $P$ for interaction $=0.68$ ). There were also no statistically significant effect modifications between tea and CRC risk by body mass index ( $P$ for interaction $=0.28)$, smoking status $(P$ for interaction $=0.94)$, alcohol consumption $(P$ for interaction $=0.72)$ or meat intake $(P$ for interaction $=0.64)$.

\section{DISCUSSION}

In this large prospective study from a CRC screening trial, we found that greater coffee and tea intakes were not associated with risk of colorectal cancer. These findings were consistent for coffee with and without caffeine and for tea irrespective of cancer site and stage or selected demographic and risk factors.

Studies of coffee intake with CRC risk are inconclusive. For the most part, significant inverse associations have been reported in case-control studies. A recent, meta-analysis of 25 case-control studies comparing the highest category of coffee intake to the none/lowest intake category reported RRs of 0.85 (95\% CI $=0.75-0.97)$ for CRC ( $\mathrm{Li}$ et al, 2012). However, these case-control studies are prone to recall bias related to dietary intake since diet intake assessment was carried out after cancer diagnosis. It is also possible that after cancer diagnosis, coffee or tea drinking habits could have changed.

Recently, the NIH-AARP Diet and Health Study, a very large cohort study of older adults (6946 cases), showed significant inverse associations between coffee and CRC risk $(\mathrm{RR}=0.80,95 \%$ $\mathrm{CI}=0.69-0.94) \quad($ Sinha et al, 2012), due largely to an inverse relationship for proximal tumors $(\mathrm{RR}=0.62,95 \% \mathrm{CI}=0.49-0.81)$. Contrary to these findings, most cohort studies have reported null associations of coffee and risk of CRC, consistent with our findings. An analysis of comparable size, the Pooling Project of Prospective Studies of Diet and Cancer, which pooled data from 13 studies, reported a null association for their highest category of coffee intake and colon cancer $(\mathrm{RR}=1.07,95 \% \mathrm{CI}=0.89-1.30)$ (Zhang et al, 2010). Also, the Pooling Project noted no risk differentials by cancer site (proximal and distal colon cancers). A 2012 metaanalysis of 16 cohort studies (10 443 cases) reported no significant association between coffee consumption and CRC risk $(\mathrm{RR}=0.94$, 95\% CI $=0.88-1.01)($ Li et al, 2012).

Most significant, inverse associations in cohort studies have been observed in subgroup analyses (typically by gender or age). The two studies that have reported significant inverse associations in women were carried out in Japanese populations (Oba et al, 2006; Lee et al, 2007), whereas a study on a Norwegian population (mostly men) only observed a statistically significant association among men younger than 65 years (Jacobsen et al, 1986). This study explored potential interactions by stratifying the cohort by several demographic and risk factors, resulting in no significant differences.

Limiting the analyses to either caffeinated or decaffeinated coffee drinkers did not alter the associations observed in the main analysis. In the United States, pooled analysis of the Nurses' Health Study and Health Professionals' Follow-Up Study also resulted in 


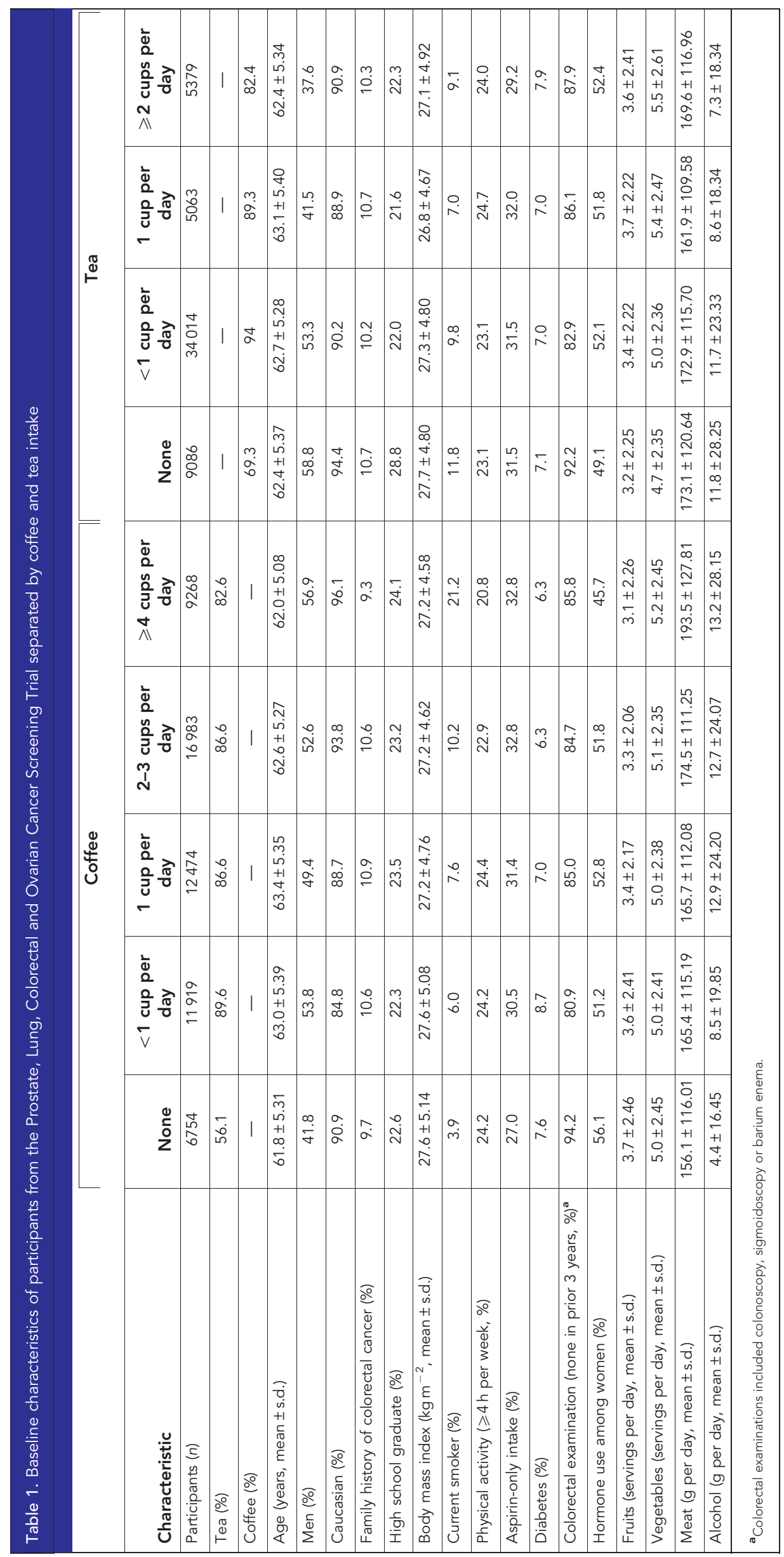




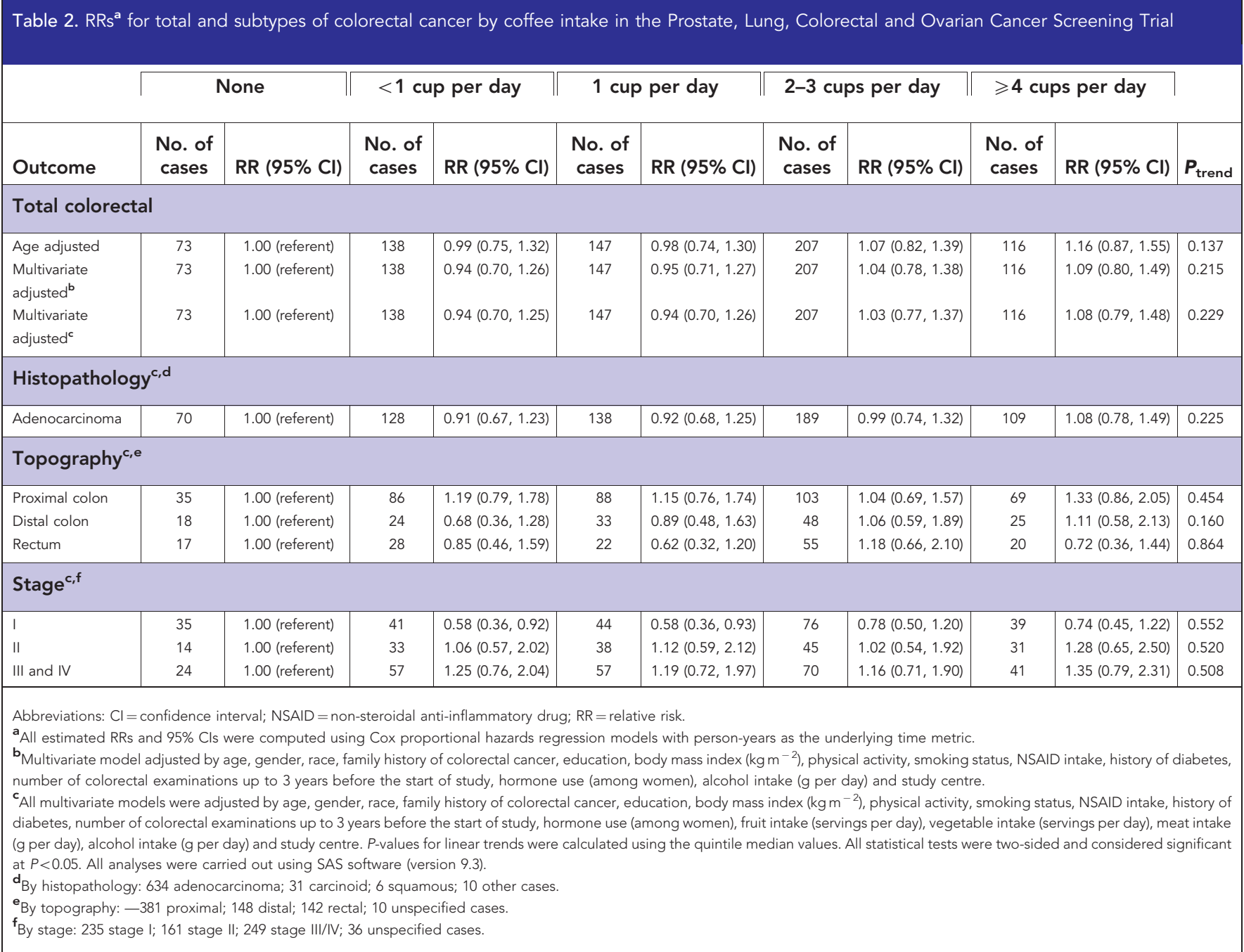

null associations for caffeinated coffee. Using $5+$ cups per day as the highest grouping of coffee intake, the RR (95\% CI) for CRC was 0.98 (0.69-1.38) (Michels et al, 2005). However, they did report a significant, inverse association between decaffeinated coffee and rectal cancer $(R R=0.48,95 \% C I=0.29-0.81)$. In this study, decaffeinated coffee was not associated with overall CRC risk and the fairly small number of rectal cases did not permit further subset analysis for decaffeinated coffee drinkers.

Ten cohort studies (Goldbohm et al, 1996; Zheng et al, 1996; Nakachi et al, 2000; Nagano et al, 2001; Terry and Wolk, 2001; Michels et al, 2005; Suzuki et al, 2005; Sun et al, 2007; Simons et al, 2010; Sinha et al, 2012) have reported null associations between tea (green, black or unspecified) and CRC risk. A meta-analysis (Sun et al, 2006) of black tea studies reported a combined RR of 1.02 $(95 \% \mathrm{CI}=0.78-1.24)$, whereas a meta-analysis (Wang et al, 2012) of green tea studies reported a combined RR of $0.90 \quad(95 \%$ $\mathrm{CI}=0.72-1.08$ ).

In contrast, a handful of cohort studies have generated significant inverse (Su and Arab, 2002; Yang et al, 2007, 2011) or positive (Heilbrun et al, 1986; Hartman et al, 1998; Terry and Wolk, 2001; Sun et al, 2007; Zhang et al, 2010; Sinha et al, 2012) associations between tea and total CRC risk, as well as within various subgroups. Significant, inverse associations between tea and CRC risk were reported in the Shanghai Women's Health Study (Yang et al, 2007) and the Shanghai Men's Health Study (Yang et al, 2011). For men, the effect was particularly profound among non-smokers for colon cancer. One possible explanation for the inconsistent findings between the Shanghai studies $v s$ this study could be cultural and lifestyle differences in the type and amount of tea consumed. Both studies excluded those who drank black tea, the type of tea more commonly consumed in western countries (Mukhtar and Ahmad, 2000; Yang et al, 2007, 2011). Although the NIH-AARP Diet and Health Study (Sinha et al, 2012) reported a null association for tea and overall CRC risk, it did find a significant, positive association with distal colon cancers. Similarly, the Pooling Project of Prospective Studies of Diet and Cancer reported a significant, positive association between tea and colon cancer (Zhang et al, 2010). However, both studies address the possibility that these findings could be due to chance.

There are several strengths of this study. First, as diet and demographic information were collected before the diagnosis of cancer in this large prospective study, differential recall bias by disease status was minimised. Second, extensive baseline and diet intake information were collected allowing for appropriate control of confounders and additional subgroup analyses. Lastly and uniquely to this study, because the majority of the cohort $(83.3 \%)$ had complete flexible sigmoidoscopy CRC screening at baseline, all participants had an equal opportunity to be screened, so any screening-related biases are unlikely to be a factor in this study. However, because our study was conducted in the screening arm of a randomised controlled trial of CRC screening, results may not be generalisable to all cases. 


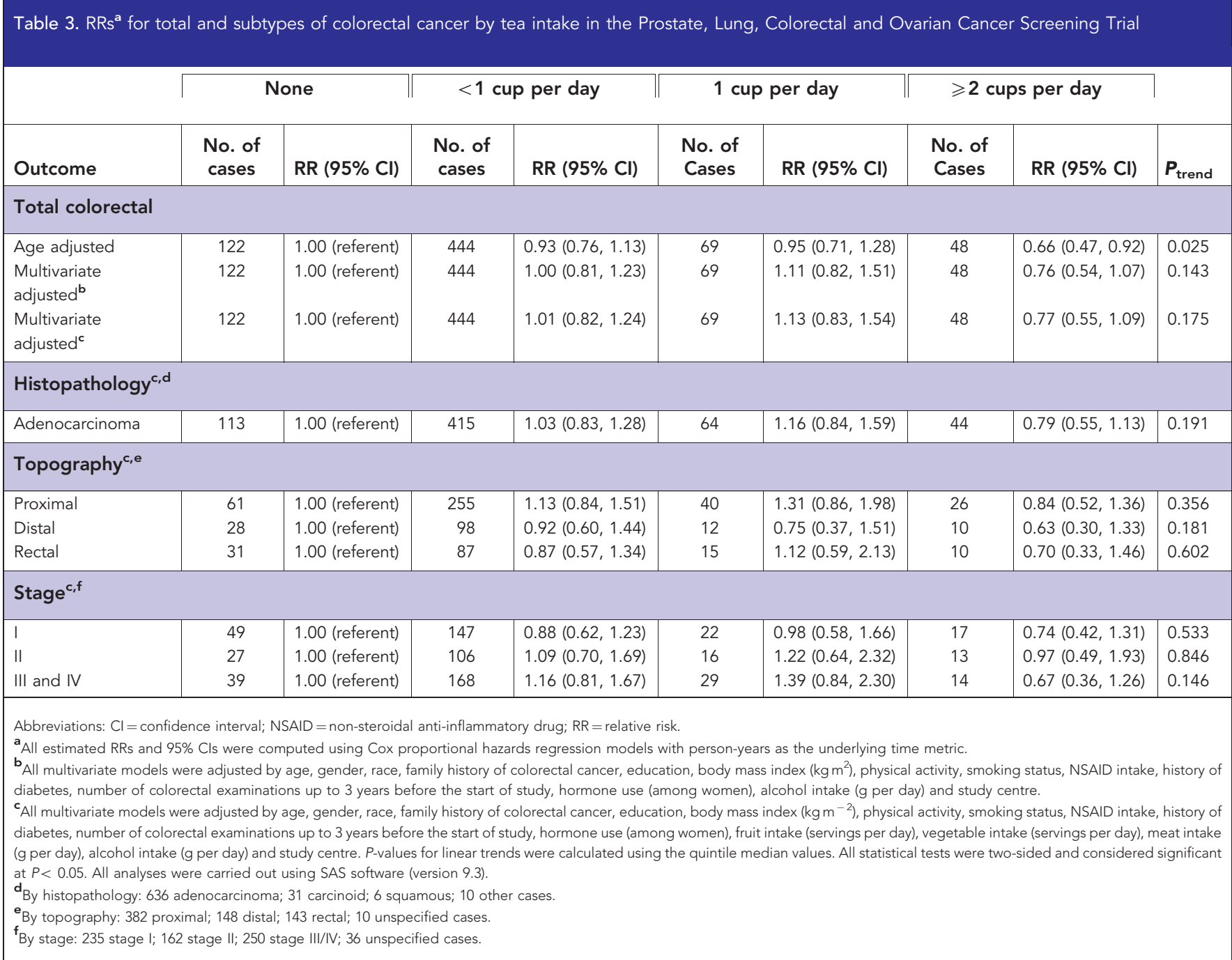

There are several limitations to this study. An inherent limitation of dietary questionnaires is their potential for nondifferential measurement error, which can bias the results towards the null (Willett, 1998). Another limitation is the natural complexity of the content of coffee and tea, which is not adequately captured by current dietary assessment methods. Furthermore, neither of the dietary questionnaires distinguished between brewing methods for coffee, which may impact its anticarcinogenic effects. Brewing methods can typically be divided into two categories, boiling and filtering. There has only been one study looking at brewing method and CRC risk, and it reported null associations (Nilsson et al, 2010). However, it is noteworthy that anticarcinogenic effects of coffee diterpenes have been reported (Cavin et al, 2002; Huber et al, 2004), the major component removed by filtering (Ruiz del Castillo et al, 1999), which happens to be the more common of the two practices among Americans. The questionnaires also did not differentiate the type of tea consumed (black or green). As black tea is more commonly consumed among Americans, combined analysis of both types of tea might mask potential tea type-specific associations. Although the main analysis had sufficient power (minimum detectable relative risk of 0.86 for coffee and CRC), the power to detect an association in specific subgroups, such as by location (proximal, distal or rectal) or coffee type (caffeinated $v s$ decaffeinated) was limited.

In conclusion, the findings of this study do not provide evidence that drinking coffee or tea is beneficial in protecting against colorectal cancer. Furthermore, the inconsistent results across all types of studies do not conclusively support the protective effects of coffee or tea against CRC and do not lead to specific recommendations for use of these beverages for CRC risk reduction.

\section{ACKNOWLEDGEMENTS}

We thank Drs Christine Berg and Philip Prorok, Division of Cancer Prevention, National Cancer Institute, the Screening Centre investigators and staff of the PLCO Cancer Screening Trial, Tom Riley and Staff, Information Management Services Inc., Barbara O'Brien and staff and Westat Inc. Most importantly, we acknowledge the study participants for their contributions to making this study possible. This work was supported by National Cancer Institute R03 CA159414, AACR Career Development Award, R01CA159036 and by the Intramural Research Program of NIH/National Cancer Institute, Division of Cancer Epidemiology and Genetics.

\section{CONFLICT OF INTEREST}

The authors declare no conflict of interest. 


\section{REFERENCES}

American Cancer Society (2011) Colorectal cancer facts and figures, 2011-2013.

Bernstein C, Holubec H, Bhattacharyya AK, Nguyen H, Payne CM, Zaitlin B, Bernstein H (2011) Carcinogenicity of deoxycholate, a secondary bile acid. Arch Toxicol 85: 863-871.

Bessler H, Salman H, Bergman M, Djaldetti M (2012) Caffeine alters cytokine secretion by PBMC induced by colon cancer cells. Cancer Invest 30: 87-91.

Block G, Hartman AM, Dresser CM, Carroll MD, Gannon J, Gardner L (1986) A data-based approach to diet questionnaire design and testing. Am J Epidemiol 124: 453-469.

Cavin C, Holzhaeuser D, Scharf G, Constable A, Huber WW, Schilter B (2002) Cafestol and kahweol, two coffee specific diterpenes with anticarcinogenic activity. Food Chem Toxicol 40: 1155-1163.

Cox DR (1972) Regression models and life-tables. J R Stat Soc Ser B 34: $187-18$.

Galeone C, Turati F, La Vecchia C, Tavani A (2010) Coffee consumption and risk of colorectal cancer: a meta-analysis of case-control studies. Cancer Causes Control 21: 1949-1959.

Goldbohm RA, Hertog MG, Brants HA, van Poppel G, van den Brandt PA (1996) Consumption of black tea and cancer risk: a prospective cohort study. J Natl Cancer Inst 88: 93-100.

Hartman TJ, Tangrea JA, Pietinen P, Malila N, Virtanen M, Taylor PR, Albanes D (1998) Tea and coffee consumption and risk of colon and rectal cancer in middle-aged Finnish men. Nutr Cancer 31: 41-48.

Heilbrun LK, Nomura A, Stemmermann GN (1986) Black tea consumption and cancer risk: a prospective study. Br J Cancer 54: 677-683.

Huber WW, Teitel CH, Coles BF, King RS, Wiese FW, Kaderlik KR, Casciano DA, Shaddock JG, Mulder GJ, Ilett KF, Kadlubar FF (2004) Potential chemoprotective effects of the coffee components kahweol and cafestol palmitates via modification of hepatic $N$-acetyltransferase and glutathione S-transferase activities. Environ Mol Mutagen 44: 265-276.

Jacobsen BK, Bjelke E, Kvale G, Heuch I (1986) Coffee drinking, mortality, and cancer incidence: results from a Norwegian prospective study. J Natl Cancer Inst 76: 823-831.

Je Y, Liu W, Giovannucci E (2009) Coffee consumption and risk of colorectal cancer: a systematic review and meta-analysis of prospective cohort studies. Int J Cancer 124: 1662-1668.

Kang NJ, Lee KW, Kim BH, Bode AM, Lee HJ, Heo YS, Boardman L, Limburg P, Dong Z (2011) Coffee phenolic phytochemicals suppress colon cancer metastasis by targeting MEK and TOPK. Carcinogenesis 32: 921-928.

Lee KJ, Inoue M, Otani T, Iwasaki M, Sasazuki S, Tsugane S (2007) Coffee consumption and risk of colorectal cancer in a population-based prospective cohort of Japanese men and women. Int J Cancer 121: 1312-1318.

Li G, Ma D, Zhang Y, Zheng W, Wang P (2012) Coffee consumption and risk of colorectal cancer: a meta-analysis of observational studies. Public Health Nutr 16: 1-12.

Merighi S, Benini A, Mirandola P, Gessi S, Varani K, Simioni C, Leung E, Maclennan S, Baraldi PG, Borea PA (2007) Caffeine inhibits adenosine-induced accumulation of hypoxia-inducible factor-1alpha, vascular endothelial growth factor, and interleukin-8 expression in hypoxic human colon cancer cells. Mol Pharmacol 72: 395-406.

Michels KB, Willett WC, Fuchs CS, Giovannucci E (2005) Coffee, tea, and caffeine consumption and incidence of colon and rectal cancer. J Natl Cancer Inst 97: 282-292.

Mukhtar H, Ahmad N (2000) Tea polyphenols: prevention of cancer and optimizing health. Am J Clin Nutr 71: 1698S-1702S; discussion 1703S-1694S.

Nagano J, Kono S, Preston DL, Mabuchi K (2001) A prospective study of green tea consumption and cancer incidence, Hiroshima and Nagasaki (Japan). Cancer Causes Control 12: 501-508.

Nakachi K, Matsuyama S, Miyake S, Suganuma M, Imai K (2000) Preventive effects of drinking green tea on cancer and cardiovascular disease: epidemiological evidence for multiple targeting prevention. Biofactors 13: 49-54.

Nilsson LM, Johansson I, Lenner P, Lindahl B, Van Guelpen B (2010) Consumption of filtered and boiled coffee and the risk of incident cancer: a prospective cohort study. Cancer Causes Control 21: 1533-1544.
Oba S, Shimizu N, Nagata C, Shimizu H, Kametani M, Takeyama N, Ohnuma T, Matsushita S (2006) The relationship between the consumption of meat, fat, and coffee and the risk of colon cancer: a prospective study in Japan. Cancer Lett 244: 260-267.

Patel R, Ingle A, Maru GB (2008) Polymeric black tea polyphenols inhibit 1, 2-dimethylhydrazine induced colorectal carcinogenesis by inhibiting cell proliferation via Wnt/beta-catenin pathway. Toxicol Appl Pharmacol 227: 136-146.

Prorok PC, Andriole GL, Bresalier RS, Buys SS, Chia D, Crawford ED, Fogel R, Gelmann EP, Gilbert F, Hasson MA, Hayes RB, Johnson CC, Mandel JS, Oberman A, O'Brien B, Oken MM, Rafla S, Reding D, Rutt W, Weissfeld JL, Yokochi L, Gohagan JK (2000) Design of the prostate, lung, colorectal and ovarian (PLCO) cancer screening trial. Control Clin Trials 21: 273S-309S.

Ruiz del Castillo ML, Herraiz M, Blanch GP (1999) Rapid analysis of cholesterol-elevating compounds in coffee brews by off-line highperformance liquid chromatography/high-resolution gas chromatography. J Agric Food Chem 47: 1525-1529.

Sengupta A, Ghosh S, Das RK, Bhattacharjee S, Bhattacharya S (2006) Chemopreventive potential of diallylsulfide, lycopene and theaflavin during chemically induced colon carcinogenesis in rat colon through modulation of cyclooxygenase- 2 and inducible nitric oxide synthase pathways. Eur J Cancer Prev 15: 301-305.

Shimizu M, Shirakami Y, Sakai H, Adachi S, Hata K, Hirose Y, Tsurumi H, Tanaka T, Moriwaki H (2008) (-)-Epigallocatechin gallate suppresses azoxymethane-induced colonic premalignant lesions in male C57BL/KsJ-db/db mice. Cancer Prev Res (Philadelphia) 1: 298-304.

Simons CC, Leurs LJ, Weijenberg MP, Schouten LJ, Goldbohm RA, van den Brandt PA (2010) Fluid intake and colorectal cancer risk in the Netherlands Cohort Study. Nutr Cancer 62: 307-321.

Sinha R, Cross AJ, Daniel CR, Graubard BI, Wu JW, Hollenbeck AR, Gunter MJ, Park Y, Freedman ND (2012) Caffeinated and decaffeinated coffee and tea intakes and risk of colorectal cancer in a large prospective study. Am J Clin Nutr 96: 374-381.

Su LJ, Arab L (2002) Tea consumption and the reduced risk of colon cancer results from a national prospective cohort study. Public Health Nutr 5: 419-425.

Subar AF, Midthune D, Kulldorff M, Brown CC, Thompson FE, Kipnis V, Schatzkin A (2000) Evaluation of alternative approaches to assign nutrient values to food groups in food frequency questionnaires. Am J Epidemiol 152: $279-286$.

Sukhthankar M, Alberti S, Baek SJ (2010) ( - )-Epigallocatechin-3-gallate (EGCG) post-transcriptionally and post-translationally suppresses the cell proliferative protein TROP2 in human colorectal cancer cells. Anticancer Res 30: 2497-2503.

Sun CL, Yuan JM, Koh WP, Lee HP, Yu MC (2007) Green tea and black tea consumption in relation to colorectal cancer risk: the Singapore Chinese Health Study. Carcinogenesis 28: 2143-2148.

Sun CL, Yuan JM, Koh WP, Yu MC (2006) Green tea, black tea and colorectal cancer risk: a meta-analysis of epidemiologic studies. Carcinogenesis 27: 1301-1309.

Suzuki Y, Tsubono Y, Nakaya N, Koizumi Y, Shibuya D, Tsuji I (2005) Green tea and the risk of colorectal cancer: pooled analysis of two prospective studies in Japan. J Epidemiol 15: 118-124.

Terry P, Wolk A (2001) Tea consumption and the risk of colorectal cancer in Sweden. Nutr Cancer 39: 176-179.

US Department of Agriculture (CSFII) (1997) Design and operation: the continuing survey of food intakes by individuals and the diet and health knowledge survey, 1994-1996. Nationwide Food Surveys Report No. 96-1, US Department of Agriculture, Agricultural Research Service.

Wang ZH, Gao QY, Fang JY (2012) Green tea and incidence of colorectal cancer: evidence from prospective cohort studies. Nutr Cancer 64: 1143-1152.

Weissfeld JL, Schoen RE, Pinsky PF, Bresalier RS, Church T, Yurgalevitch S, Austin JH, Prorok PC, Gohagan JK (2005) Flexible sigmoidoscopy in the PLCO cancer screening trial: results from the baseline screening examination of a randomized trial. J Natl Cancer Inst 97: 989-997.

Willett W (1998) Nutritional Epidemiology. Oxford University Press.

Willett WC, Sampson L, Stampfer MJ, Rosner B, Bain C, Witschi J, Hennekens CH, Speizer FE (1985) Reproducibility and validity of a semiquantitative food frequency questionnaire. Am J Epidemiol 122: 51-65. 
Xu M, Bailey AC, Hernaez JF, Taoka CR, Schut HA, Dashwood RH (1996) Protection by green tea, black tea, and indole-3-carbinol against 2-amino-3-methylimidazo[4,5-f]quinoline-induced DNA adducts and colonic aberrant crypts in the F344 rat. Carcinogenesis 17: $1429-1434$

Yang G, Shu XO, Li H, Chow WH, Ji BT, Zhang X, Gao YT, Zheng W (2007) Prospective cohort study of green tea consumption and colorectal cancer risk in women. Cancer Epidemiol Biomarkers Prev 16: 1219-1223.

Yang G, Zheng W, Xiang YB, Gao J, Li HL, Zhang X, Gao YT, Shu XO (2011) Green tea consumption and colorectal cancer risk: a report from the Shanghai Men's Health Study. Carcinogenesis 32: 1684-1688.

Zhang X, Albanes D, Beeson WL, van den Brandt PA, Buring JE, Flood A, Freudenheim JL, Giovannucci EL, Goldbohm RA, Jaceldo-Siegl K, Jacobs EJ, Krogh V, Larsson SC, Marshall JR, McCullough ML, Miller AB,
Robien K, Rohan TE, Schatzkin A, Sieri S, Spiegelman D, Virtamo J, Wolk A, Willett WC, Zhang SM, Smith-Warner SA (2010) Risk of colon cancer and coffee, tea, and sugar-sweetened soft drink intake: pooled analysis of prospective cohort studies. J Natl Cancer Inst 102: 771-783.

Zheng W, Doyle TJ, Kushi LH, Sellers TA, Hong CP, Folsom AR (1996) Tea consumption and cancer incidence in a prospective cohort study of postmenopausal women. Am J Epidemiol 144: 175-182.

This work is published under the standard license to publish agreement. After 12 months the work will become freely available and the license terms will switch to a Creative Commons AttributionNonCommercial-Share Alike 3.0 Unported License. 